\title{
Retroperitoneal Mass: Lymphoma as Differential Diagnosis To Retroperitoneal Fibrosis. Case Report.
}

\section{Madalina Nussberger ( $\nabla$ madalinarotaru88@yahoo.com )}

Cantonal Hospital Sankt Gallen: Kantonsspital Sankt Gallen

Olaf Kim

Cantonal Hospital Sankt Gallen: Kantonsspital Sankt Gallen

\section{Sergio Cogliatti}

Cantonal Hospital Sankt Gallen: Kantonsspital Sankt Gallen

Thomas Neumann

Cantonal Hospital Sankt Gallen: Kantonsspital Sankt Gallen

\section{Research Article}

Keywords: retroperitoneal mass, retroperitoneal fibrosis, lymphoma , case report

Posted Date: July 19th, 2021

DOI: https://doi.org/10.21203/rs.3.rs-536375/v1

License: (c) (1) This work is licensed under a Creative Commons Attribution 4.0 International License. Read Full License

Version of Record: A version of this preprint was published at SN Comprehensive Clinical Medicine on January 7th, 2022. See the published version at https://doi.org/10.1007/s42399-021-01106-9. 


\title{
Retroperitoneal mass: lymphoma as differential diagnosis to retroperitoneal fibrosis. Case report.
}

\author{
Madalina Nussberger * a, Olaf Chan-Hi Kim ${ }^{b}$, Sergio Cogliatti ${ }^{c}$, Thomas Neumann ${ }^{a}$ \\ a Department of Rheumatology and Immunology, Cantonal Hospital St. Gallen, Switzerland \\ ${ }^{\mathrm{b}}$ Department of Radiology, and nuclear medicine Cantonal Hospital St. Gallen, Switzerland \\ ${ }^{c}$ Institute of Pathology, Cantonal Hospital St. Gallen, St. Gallen, Switzerland \\ Corresponding Author: \\ Madalina Nussberger \\ Department of Rheumatology and Immunology, Cantonal Hospital St. Gallen \\ Rorschacher Strasse 95, 9007 St. Gallen, Switzerland \\ E-mail: madalinarotaru88@yahoo.com
}

\begin{abstract}
Background

The nature of a solid retroperitoneal mass can either be autoimmune or neoplastic and should be investigated by imaging and histology. The spectrum of differential diagnoses includes primarily retroperitoneal fibrosis and retroperitoneal tumors. Because treatments of retroperitoneal fibrosis and retroperitoneal tumors vary substantially, accurate and early diagnosis is highly relevant.
\end{abstract}

\section{Case presentation}

We present a case of a fifty-four-year old woman that was admitted to our hospital with stroke like symptoms. Suspecting a vasculitis, magnet resonance imaging of the head and abdomen were done, which revealed a circular enhancement of a cranial vessel as well as retroperitoneal and peri-aortic masses. Concerning this specific radiographic patterns, an autoimmune process like retroperitoneal fibrosis was hypothesized. Steroid treatment did not lead to significant remission though. On re-evaluation of the mass because of refractory disease, fine needle aspiration did not show malignant cells while surgery and histologic assessment revealed neoplastic lymphoproliferation. The final diagnosis was a non- Hodgkin B- Cell Lymphoma. Chemo- and Immunotherapy was initiated. A control abdominal computer tomography was performed which revealed a significant remission of the retroperitoneal mass. 


\section{Conclusion}

Initially, the retroperitoneal mass was highly suspicious for RF. The results of imaging can be useful, however, histology should always be taken into consideration when there is an uncertain clinical presentation. Without histology we would have missed a non-Hodgkin B-Cell Lymphoma in this case. Minimal invasive techniques like fine needle aspiration may be practical but can demonstrate false negative results.

Keywords: retroperitoneal mass, retroperitoneal fibrosis, lymphoma, case report 


\section{Background}

Retroperitoneal masses can be a diagnostic challenge for clinicians because radiologic findings may suggest a number of possible diagnoses. The main differential diagnosis is retroperitoneal fibrosis (RF) which consists of chronic soft tissue fibrosis in the retroperitoneum, often leading to compression of retroperitoneal organs (i.e. obstructive mega-ureter/hydronephrosis and renal failure) and/or compression of veins, arteries, and lymphatic vessels leading to edema, thrombotic disease, hydrocele, and claudication (1). In the retroperitoneum, lymphoma is the most frequent malignant tumor after retroperitoneal sarcoma $(2,3)$, but metastases originating from germ cell tumors or other epithelial tumors may also be seen in the retroperitoneal space (2). However, the retroperitoneal space is uncommon as the primary site and exclusive manifestation of a malignant lymphoma (3-5). Retroperitoneal lymphomatous involvement may be secondary to continuous spread from abdominal lymph nodes (5). Radiologic imaging may permit first differential diagnostic triage, particularly with respect to RF (6), but this needs to be confirmed histologically (1).

We present a case of a 54-year-old female patient with an unclear retroperitoneal mass not responsive to steroid treatment.

\section{Case Presentation}

A 54-year-old woman with a medical history of unprovoked lung and brain embolism underwent further investigation. She presented with paresthesia and disabling weakness of the right hand that had persisted for three days. Furthermore, she had been suffering from daily headache attacks for three weeks. At clinical presentation, the patient was afebrile, hypertensive $(167 / 78 \mathrm{mmHg}$ ), normocard (HR $71 \mathrm{bpm}$ ), had a body weight of $90 \mathrm{~kg}$ (calculated BMI of 29.4 $\mathrm{kg} / 2 \mathrm{~m}^{2}$ ) and a normal oxygen saturation (95\%) breathing ambient air. Examination of her lungs and heart were inconspicuous. Neurological findings showed paresis of the right finger movements (i.e. extension, flexion, spreading of the fingers, ab- and adduction, as well as opposition of the thumb). The remainder of the examination was normal.

Routine laboratory testing revealed normal C-reactive protein (CRP) levels and blood sedimentation rate (BSR). Creatinine was mildly elevated $(111 \mu \mathrm{mol} / \mathrm{L}$ (normal values $<95$ $\mu \mathrm{mol} / \mathrm{L}$ ); calculated GFR [Cockcroft-Gault] $73 \mathrm{~mL} / \mathrm{min}$, reference value $>70 \mathrm{~mL} / \mathrm{min}$ ). She had normal values for liver enzymes, protein electrophoresis, blood count, and urine analysis. Autoimmune serology was normal for antinuclear antibodies (ANA) and antineutrophil cytoplasmatic antibodies (ANCA), and there was no elevation of antiphospholipid antibodies. Testing for hereditary or acquired thrombophilia was negative. Magnetic resonance imaging 
(MRI) of the head showed multiple cerebral infarctions, but the most striking finding was an isolated circular enhancement of the left internal carotid artery (ICA) suggesting vasculitis. Examinations of the heart (i.e. $24 \mathrm{~h}$ rhythmic analysis and ECG) were normal. Abdominal sonography demonstrated a shrunken unilateral kidney. Finally, MRI revealed extensive periaortal and left para-aortal soft tissue masses along the left iliacal interna artery, measuring $6.5 \mathrm{x}$ $2.5 \mathrm{~cm}$. Two computer tomography (CT)-guided fine needle aspirations (FNA) of the retroperitoneal mass were performed without any conclusive cytologic or molecular genetic results, neither for retroperitoneal fibrosis (Morbus Ormond; CD 38 and IgG4 negative) nor for malignancy.

Initially assuming idiopathic retroperitoneal fibrosis, we started prednisone treatment that proved unsuccessful. Follow-up CT of the head 6 months later showed a decline in vessel caliber and vessel wall enhancement of the left ICA compared to initial findings, while CT follow-up of the abdomen 5 months later demonstrated progression of the retroperitoneal mass. Tissue samples from the left iliacal region obtained by diagnostic Da-Vinci laparoscopy were compatible with malignant B-cell lymphoma, both morphologically and immunohistochemically. To verify this finding, we performed immunoglobulin heavy-chain gene rearrangement by polymerase chain reaction (PCR) that yielded clonality in frameworks 2 and 3 (Fig. 3). Chemo - and Immunotherapy with R-Bendamustin was carried out for 6 months. Eight months after diagnosis, we observed a partial response with downsizing of the retroperitoneal mass in response to chemotherapy. The therapy was well tolerated by the patient who remained in good condition and regularly attended the planned follow-up visits. Currently, maintenance therapy with rituximab is planned for two additional years to optimize progression-free survival. NHL has a good prognosis with modern treatment, the estimated five years survival is considered to be over $85 \%$ percent (7).

\section{Discussion and Conclusion}

This case report illustrates the challenges in connection with the diagnosis of a retroperitoneal mass. Initially, we diagnosed the mass as RF, but subsequent histologic work-up confirmed the presence of B-cell lymphoma.

Initial neurological presentation with stroke due to multiple cerebral vascular irregularities led to the assumption of vasculitis. Further diagnostic steps to confirm vasculitis consisted of angio-MRI of the entire aorta and proximal vessels, which revealed an unknown retroperitoneal mass interpreted as RF. Clinical onset of RF may be asymptomatic or may mimic lymphoproliferative diseases (8). The main symptoms of RF are compression of retroperitoneal organs (i.e. obstructive mega-ureter/hydronephrosis and renal failure) and other organs. Additional 
symptoms include non-specific constitutional symptoms such as fatigue, anorexia, and weight loss (1).

In our case, FNA failed to achieve a conclusive diagnosis. However, the MRI pattern appeared to be characteristic of RF. In line with other cases described in the literature, we noted a peri-aortic mass (Figures 1 and 2) with pelvic extension and associated ureteral encasing (9). Glucocorticoid treatment did not lead to remission. CT-follow-up of the abdomen 5 months later showed progression of the mass with no systemic inflammatory signs, which made us reconsider the diagnosis.

Inflammatory markers such as erythrocyte sedimentation rate (ESR) and CRP are elevated in more than $50 \%$ of RF patients (10). In approximately one third of cases, RF is secondary to other causes (i.e. infection, autoimmune disease, medications, radiotherapy, surgery, malignant lymphoma, or other cancers found in about $8 \%$ of cases). Two thirds of RF cases are considered to be idiopathic (6). The IgG4-related disease (IgG4-RD) leading to RF is a fibro-inflammatory condition characterized by a dense lymphoplasmacytic infiltrate rich in IgG4-positive plasma cells and involving sclerosis including lymph nodes (6). In their case report, Sato et al. postulated a neoplastic potential of IgG4-producing cells leading to lymphoma (11), but our patient had no serological elevation of IgG4. Histologic findings in biopsies obtained laparoscopically demonstrated clonal rearrangement of immunoglobulin heavy chain genes (Figure 3: frameworks 2 and 3) thus confirming B-cell lymphoma.

Retrospectively, embolism could have been interpreted as part of a paraneoplastic syndrome, and vasculitis could have been understood as secondary manifestation of extra-nodal lymphoma. Lymphoma predominantly occur in suprarenal locations with perirenal extension and tend to be associated with retroperitoneal lymph node enlargement, resulting in larger contrast-enhanced areas (9). These typical findings were not present in our patient. Further anterior displacement of the aorta and lateral displacement of the ureter seem to occur more often in malignancy than (idiopathic) RF (9).

Early in our diagnostic work-up, FNA failed to rule out malignancy or RF. FNA has a limited role in the diagnosis of lymphoma because the samples obtained by FNA do not provide the anatomic details derived from larger, intact nodal specimens (3). Guo et al. compared 68 samples obtained by radiologically guided FNA with 36 biopsies of pelvic and retroperitoneal masses. The authors calculated a sensitivity of $90.2 \%$ and specificity of $100 \%$ for FNA (12). Another study in 167 FNA samples obtained by ultrasonic guidance found a sensitivity of $86 \%$ and specificity of $100 \%$ in terms of differentiating between malignant and benign lesions of the retroperitoneum (13). 
After establishing the diagnosis of malignant B-cell lymphoma, the therapeutic regimen consisted of 6 cycles of R-Bendamustin to prevent compression of the ureter as well as further retroperitoneal spread. The patient is receiving rituximab maintenance therapy for 2 years. Interestingly, cerebral vascular lesions had normalized 6 months after start of prednisone treatment as seen in the follow-up MRI of the head. This may have been due to glucocorticoid treatment.

In conclusion, lymphoma mimicked RF in terms of imaging characteristics and vasculitis in our patient. Retrospectively, we should have aimed for histologic evidence already initially and not only in the context of refractory disease. Diagnostic minimally invasive procedures to obtain biopsies of deep masses can be helpful because the volume of extracted tissue by FNA tends to be insufficient for further investigations (i.e. microscopic examination, flow cytometry, immunophenotyping, and molecular analysis) (3).

\section{List of abbreviations}

ANA: Antinuclear antibody

ANCA: Antinulear cystoplasmatic antibody

Bpm: Beats per minute

BMI: Body mass index

CRP: C-reactive protein

$\mathrm{CT}$ : Computed tomography

CD: Cluster of differentiation or cluster of designation

ECG: Electrocardiogram

ESR: Erythrocyte sedimentation rate

FNA: Fine needle aspiration

GFR: Glomerular filtrate rate

HR: Heart rate

ICA: Internal carotid atery

i.e.: That is (id est, latin)

IgG: Immunoglobulin G

IgG4: Immunoglobulin G4 
IgG4-RD: Immunoglobulin G4-related disease

Kg: Kilogram

RF: Retroperitoneal fibrosis

mmHg: millimeters of mercury

MRI: Magnetic resonance imaging

NHL: non-Hodkgin Lymphoma

PCR: Polymerase chain reaction

R-Bendamustin: Rituximab-Bendamustin

\section{Declarations}

\section{Ethics approval and consent to participate}

The authors declare no ethical conflict.

\section{Consent for publication}

Written informed consent was obtained from the patient for publication of this case report and any accompanying images. A copy of the written consent is available for review by the Editor of this journal.

\section{Availability of data and materials}

The datasets used and/or analysed during the current study are available from the corresponding author on reasonable request.

\section{Competing interests}

The authors declare no competing interests.

\section{Funding Sources}

No specific funding was necessary for the realization of this publication.

\section{Author Contributions}

MN was responsible for writing the article. OK and SG contributed radiologic and histologic images and interpretation. TN supervised and reviewed the entire process. All authors read and approved the final manuscript.

\section{Acknowledgements}

Not applicable. 


\section{References}

1. Wan N, Jiao Y. Non-Hodgkin lymphoma mimics retroperitoneal fibrosis. Case Rep. 2013; Doi: 10.1136/bcr-2013-010433.

2. Sassa N. Retroperitoneal tumors: Review of diagnosis and management. Int J Urol. 2020; Doi: 10.1111/iju.14361.

3. Chen LL, Kuriakose P, Hawley RC, Janakiraman N, Maeda K. Hematologic Malignancies With Primary Retroperitoneal Presentation: Clinicopathologic Study of 32 Cases. Arch Pathol Lab Med. 2005; Doi: 10.1043/1543-2165

4. Fulignati C. An uncommon clinical presentation of retroperitoneal non-Hodgkin lymphoma successfully treated with chemotherapy: A case report. World J Gastroenterol. 2005; Doi: 10.3748/wjg.v11.i20.3151.

5. CONSTANTIN A, TĂNASE AD. A Primary Retroperitoneal Diffuse Large B-Cell Lymphoma: A Challenging Diagnosis. Curr Health Sci J. 2018; Doi: 10.12865/CHSJ.44.04.12.

6. Alvarez Argote J, Bauer FA, Posteraro AF, Dasanu CA. Retroperitoneal fibrosis due to B-cell non-Hodgkin lymphoma: Responding to rituximab! J Oncol Pharm Pract. 2016; Doi: 10.1177/1078155214543279.

7. Siegel RL, Miller KD, Jemal A. Cancer statistics, 2017. CA Cancer J Clin. Januar 2017; Doi: 10.3322/caac. 21387

8. Sica A, Casale B, Spada A, Teresa Di Dato M, Sagnelli C, Calogero A, u. a. Differential diagnosis: retroperitoneal fibrosis and oncological diseases. Open Med. 2019; Doi: 10.1515/med2020-0005.

9. Rosenkrantz AB, Spieler B, Seuss CR, Stifelman MD, Kim S. Utility of MRI Features for Differentiation of Retroperitoneal Fibrosis and Lymphoma. Am J Roentgenol. 2012; Doi: 10.2214/AJR.11.7822.

10. Urban ML, Palmisano A, Nicastro M, Corradi D, Buzio C, Vaglio A. Idiopathic and secondary forms of retroperitoneal fibrosis: A diagnostic approach. Rev Médecine Interne. 2015; Doi: 10.1016/j.revmed.2014.10.008.

11. Sato $\mathrm{Y}$, Takata $\mathrm{K}$, Ichimura $\mathrm{K}$, Tanaka T, Morito $\mathrm{T}$, Tamura $\mathrm{M}$, u. a. IgG4-producing marginal zone B-cell lymphoma. Int J Hematol. 2008; Doi: 10.1007/s12185-008-0170-8.

12. Guo Z, Kurtycz DFI, De Las Casas LE, Hoerl HD. Radiologically guided percutaneous fineneedle aspiration biopsy of pelvic and retroperitoneal masses: A retrospective study of 68 cases. Diagn Cytopathol. 2001; Doi: 10.1002/dc.2000.

13. ISSN international centre: 0012-0472. http://www.issn.org (2006). Accessed 1946. 


\section{Figure legends}

Fig. 1: Axial T1 weighted fat suppressed image without contrast agent of the abdominal aorta with a retroperitoneal left sided periaortic mass (indicated by arrows).

Fig. 2: Axial T1 weighted fat suppressed image with contrast agent enhancing periaortic mass (indicated by arrows)

Fig. 3: Clonal rearrangement of the immunoglobulin heavy-chain gene locus (IGH) in framework II (FR2) and III (FR 3) next to a polyclonal rearrangement pattern in framework I (FR 1) revealed by Multiplex-PCR (IdentiClone IGH Gene Clonality Assay) and fragment analysis. 


\section{Figures}

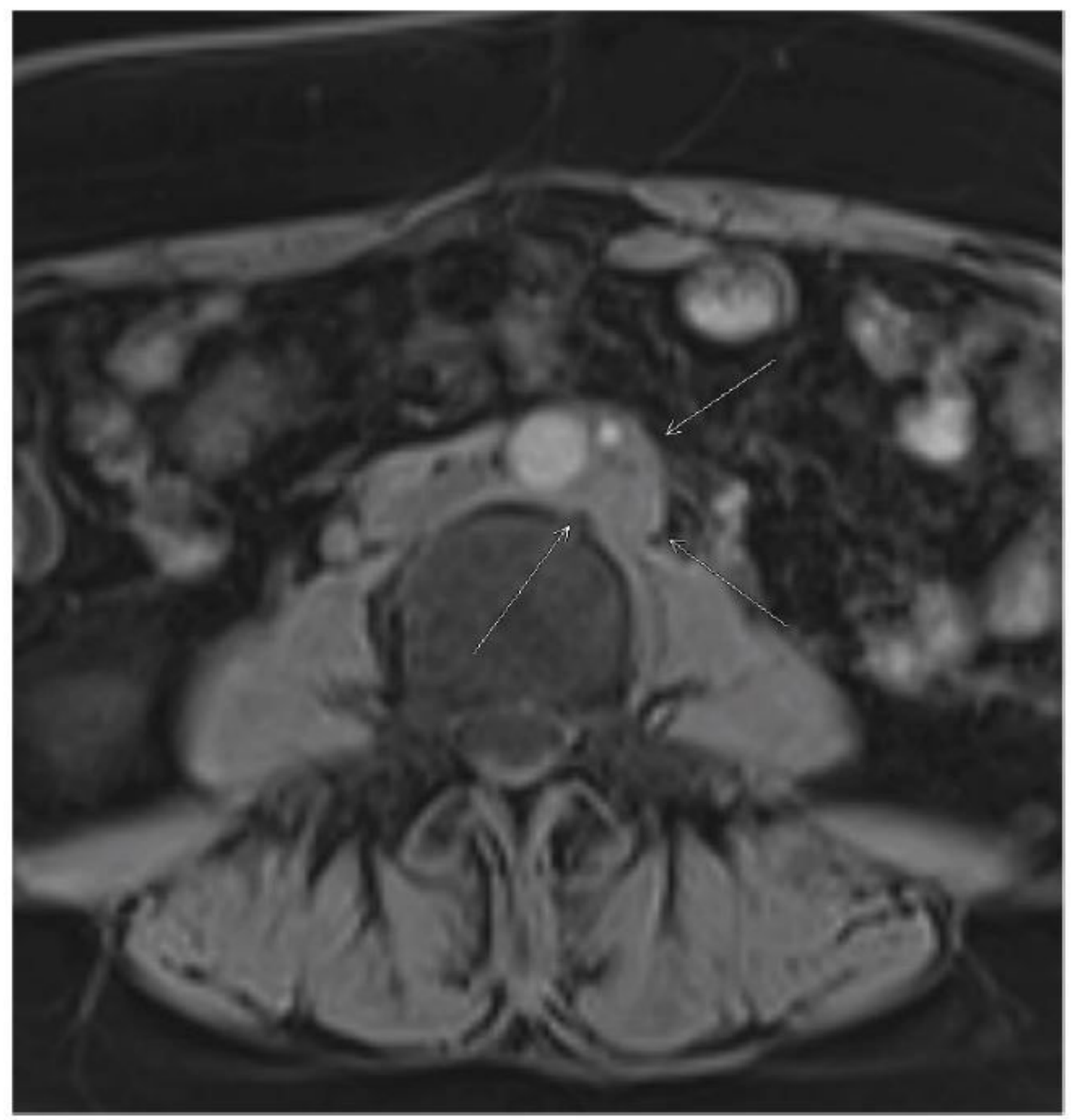

Fig. 1

\section{Figure 1}

Axial T1 weighted fat suppressed image without contrast agent of the abdominal aorta with a retroperitoneal left sided periaortic mass (indicated by arrows). 


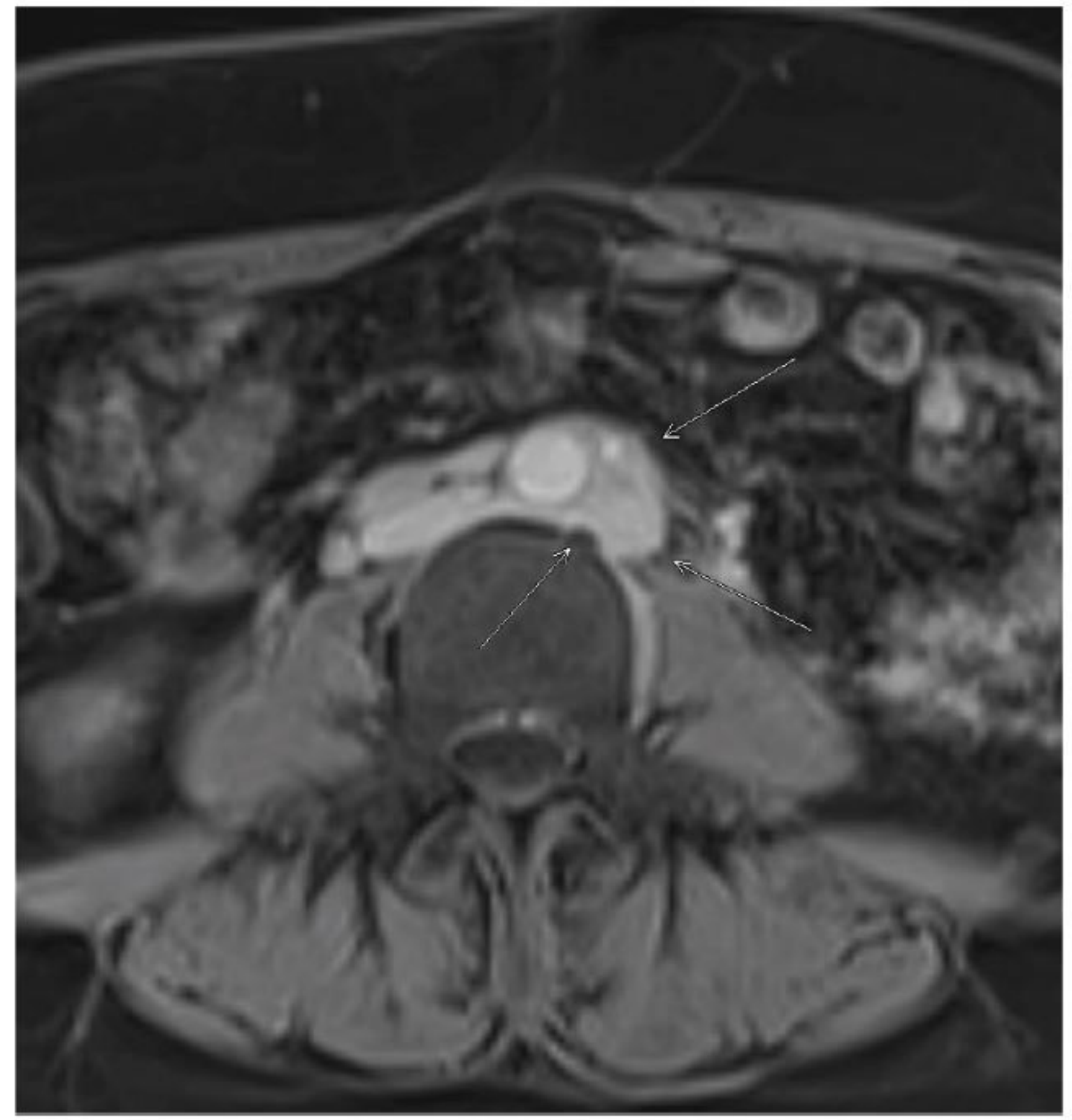

\section{Fig. 2}

\section{Figure 2}

Axial T1 weighted fat suppressed image with contrast agent enhancing periaortic mass (indicated by arrows) 
Fig. 3
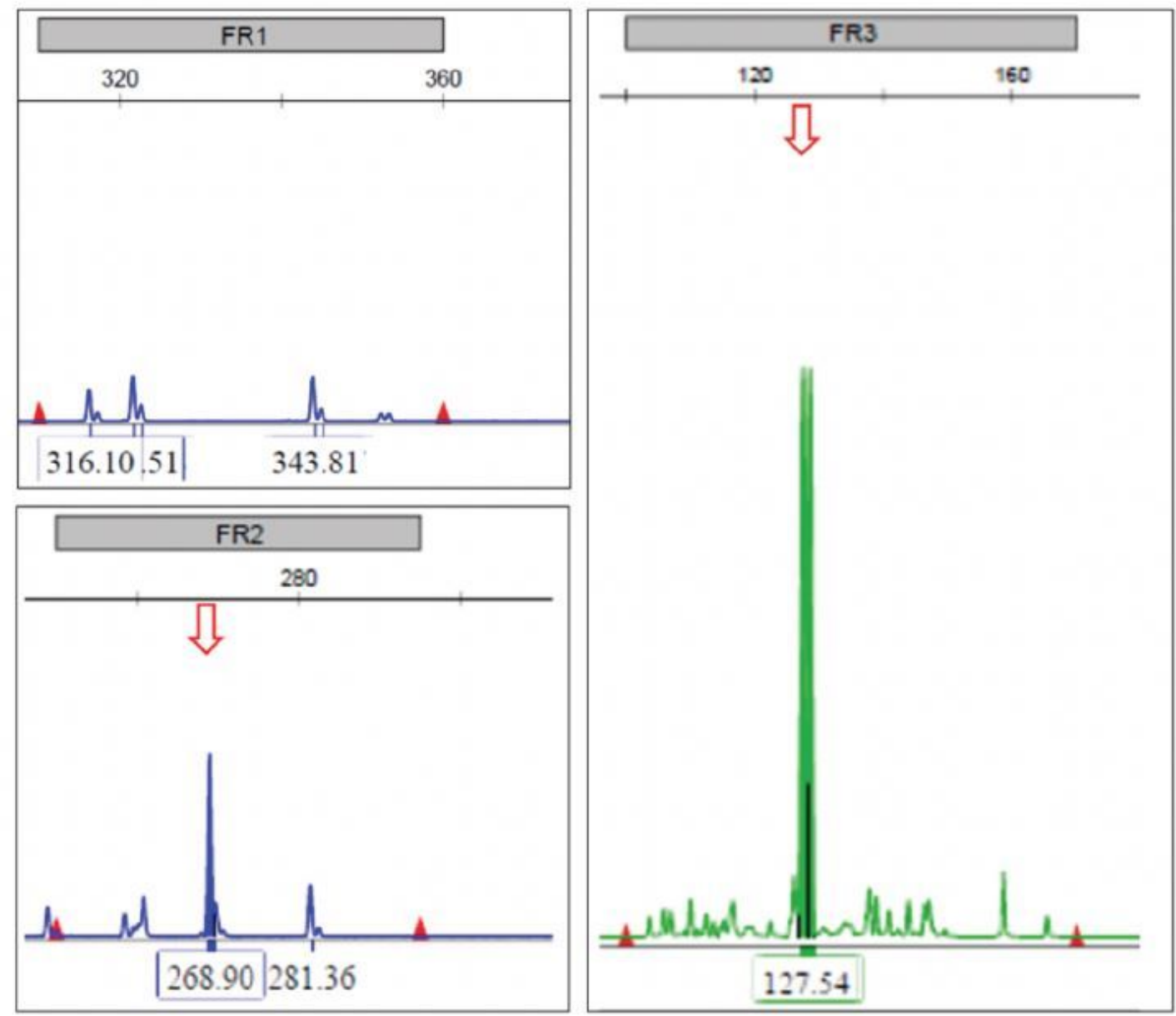

Figure 3

Clonal rearrangement of the immunoglobulin heavy-chain gene locus (IGH) in framework II (FR2) and III (FR 3) next to a polyclonal rearrangement pattern in framework I (FR 1) revealed by Multiplex-PCR (IdentiClone IGH Gene Clonality Assay) and fragment analysis.

\section{Supplementary Files}

This is a list of supplementary files associated with this preprint. Click to download.

- ChecklistCARE.pdf 